\title{
L'HISTOIRE SOCIALE DE L'ART: UN ALIBI? *
}

Nicos Hadjinicolaov

Permettez--moi dans cette communication, qui porte un titre assez influencé par des romans policiers, de jouer le jeu jusqu'au bout et, tel un imitateur maladroit de Hercule Poirot, de procéder de la manière suivante : d'abord de vous faire part de quelques observations (certes non point "innocentes" ou "objectives" puisqu'elles découlent du partipris qui m'a permis de les faire, mais néanmoins observations de faits que je crois indéniables, qui seront pour moi les "indices", "Ia piste" je voudrais suivre) puis de vous proposer quelques reflexions plus générales sur le sujet qui me préoccupe.

\section{I}

La Bibliothèque Nationale de France possède un "fichier matières" (catalogue par "mots typiques" était son titre initial) à partir de 1882, c'est-à-dire depuis un siècle. Il est divisé en six tranches : 1882-1894, 1894-1925, 1925-1935, 1936-1959, 1960-1979, et depuis 1980. Nous ne possedons pas d'études sur les principes de classification du catalogue "matières" et leur histoire. Toujours est-il que ce fichier, au-delà des services inmédiats qu'il rend aux chercheurs, est non seulement l'illustration vivante de l'historicité des catégories de pensée des classificateurs mais aussi de l'attraction (changeante) qu'exercent dans le temps certains sujets-matières. Une étude quantitative de ce fichier peut donc être, en dépit des lacunes existantes et les partis pris ou même les fautes indiscutables des classiticateurs, tout à fait instructive. Cherchons sous "histoire sociale". Entre 1882 et 1894 il n'y a rien entre "histoire sainte" et "histoire universelle". De 1894 à 1925 il y a deux fiches (Les "découvertes d'histoire sociale" par Georges d'Avenel et la publication periodique allemande "Sozialgeschichtliche Forschungen"). De 1925 à 1935 on trouve entre "l'histoire sainte" et "l'histoire universelle" "l'histoire sans issue", mais aucune trace de l'histoire sociale. Mème chose pour les années 1936 à 1959. Et puis le "take-off" se dessine : de 1960 à 1979

* Communication au colloque "Théories et applications de l'histoire sociale de l'art", Musée des Beaux-Arts, Chartres, 10-11 décembre 1983. 
il y a 4 fiches sur "histoire sociale". Depuis 1980 (c'est-à-dire depuis trois ans) il y a déjà 18 fiches.

Les comparaisons entre l'attraction qu'exercent certaines notions sont toujours hasardeuses. Mais on pourrait peut-être soutenir l'argument que la fortune de "l'histoire sociale" est l'inverse de celle d'une autre notion que nous connaissons bien tous, que nous utilisions il n'y a pas très longtemps plusieurs fois par semaine, mais qui, probablement, n'a pas été employée une seule fois par aucun de nous ici présents durant toute l'année 1983: je pense au "structuralisme". Pourtant il partage avec l'histoire sociale les mêmes débuts modestes : Si la notion de "struc. ture" apparaît d'une manière croissante depuis 1894 jusqu'à 1959 dans les travaux qui traitent presque exclusivement de la biologie ou des mathématiques, le terme "structuralisme" apparaît pour la première fois dans les fichiers de la Bibliothèque Nationale depuis 1960, pour battre tous les records avec 126 fiches entre 1960 et 1979 (126 au lieu de 4 sur "histoire sociale" !!), subdivisées en catégories plus étroites ("structuralisme phénoménologique", "structuralisme soviétique", "structuralisme linguistique", etc.). Par contre depuis 1980 il n'y a, en tout, que dix fiches sur "structuralisme", presque la moitié de celles qui existent sur "histoire sociale".

"Hasardeuse", cette comparaison entre l'attraction inégale qu'excercent deux notions sur nous et nos contemporains ${ }^{1}$ est aussi "tendancieuse" : au delà des avantages qu'a toute historicisation de notre présent (non pas sous forme d'une objectivation de type positiviste mais comme conception du présent en tant que résultat de tendances conflictuelles ayant une dynamique propre), il est évident que le choix de "structuralisme" comme terme comparatif, me permet d'insinuer que la gloire actuelle de l'histoire sociale sera aussi passagère que celle du structuralisme. Je crois pourtant, sans vouloir ouvrir un débat là-dessus, qu'il y a quelque chose de plus dans cette juxtaposition que ma mauvaise foi. "Structuralisme" est certainement une notion clé des sciences sociales des années '70 et surtout de années '60. "Histoire sociale" ne joue pas encore un rôle aussi stratégique, mais son attraction croissante indique une tendance nette dans cette direction.

1 A condition bien entendu, que les fichiers de la Bibliothèque Nationale soient réprésentatifs poux ce qui se passe dans le monde de l'édition, du moins en France, ce dont j'en suis convaincu. 
Cette montée, certes "résistible", de l'histoire sociale se fait remarquer aussi plus prés de nous, et pas seulement dans les fichiers d'une bibliothèque, en pleine pratique quotidienne de l'histoire de l'art, où "l'histoire sociale de l'art" est au centre des débats théoriques et méthodologiques. Il suffirait peut-être de mentionner le colloque sur "l'histoire sociale de l'art et de la littérature" qui sera organisé au mois d'avril 1984 par Hans-Robert Jauss, le colloque de Pise "Juger et classer : pour une histoire sociale de la perception artistique", pour ne pas parler des projets de l'Ulmer Verein ou de l'Université de Lausanne sur le même sujet; enfin la place qu'accordent les Actes de la Recherche en Sciences Sociales, depuis leurs débuts, à la question.

Troisième observation liant un peu les deux précédentes : l'origine du "boom" actuel est récente. Quand quelqu'un, avec quand même une formation d'historien d'art aussi, publiait il y a trente ans une "histoire sociale de l'art et de la littérature" (j'ai pu le constater gràce à Michael Orwicz qui m'a permis de consulter son dossier sur la réception du livre de Hauser en Allemagne), il était compiètement ignoré par les historiens d'art. Un seul historien d'art de langue allemande (Klaus Lankheit) a écrit un compte-rendu sur le livre de Hauser et cela a été publié dans la revue de sociologie de Cologne (la Kölner Zeitschrift für Soziologie)! Ayant été, peu après, étudiant d'histoire de l'art à l'université de Munich je me rappelle avec quel dédain on parlair de Hauser dans l'Institut d'histoire de l'art. C'était pratiquement au même moment que Pierre Francastel faisait à Paris l'éloge des éditeurs français pour ne pas avoir publié Hauser en français!

Est-ce que je généralise abusivement?

Cela était-ce peut-être dû aux valeurs et surtout aux faiblesses du seul livie de Hauser? Pourtant, si l'on jette un coup d'oeil à des travaux. considerés (à tort ou à raison) comme ayant une orientation semblable (que ce soit de Klingender ou de Antal) on constatera que les réactions se recoupent. La profession, l'histoire de l'art, c'est-à-dire l'histoire de l'art dominante qui exerce en cette période un monopole quasi absolu dans plusieurs pays, refuse de prendre sériusement en compte les ouvrages 
"d'histoire sociale" ou de "sociologie" de l'art, soit parce qu'ils sont vulgaires et simplistes, soit (dans le meilleur des cas) parce qu'ils n'ont rien à voir avec l'histoire de l'art proprement dite.

Quittons provisoirement "l'histoire sociale de l'art" et essayons d'observer ce qui se passe en "histoire sociale" tout court.

Ici il y a d'abord des traditions importantes. Je mentionne en passant trois périodiques qui ont eu l'histoire sociale au centre de leurs préoccupations, même si ce n'était pas toujours affiché dans leur titre: la Vierteljahrschrift für Sozial- und Wirtschaftsgeschichte, le Journal of Social History et les Annales.

Ici il y a ensuite une reflexion plus ou moins constante sur ce qu'est "l'histoire sociale". Les tentatives de définition, de délimitation, de mise en question, s'enchainent. Observons un peu ce qui se passe dans l'historiographie française. Le Colloque de Saint-Cloud de l'Ecole Normale en 1965 sur "L'Histoire Sociale" 2 me paraît à cet égard représentatif. Les historiens parmi vous pourraient-ils me permettie de résumer les tendances qui se sont dessinées à ce colloque, quant à la nature, quant à l'objet, quant à la finalité de l'histoire sociale?

J'en vois trois. Il y a une tendance qui nie tout simplement que l'histoire sociale soit une discipline ayant un objet propre. Je cite par exemple Maurice Crubellier: "Enfin le domaine de l'histoire sociale reste mal défini. Peut-être même n'en a-t-elle point qui lui soit propre (...) Plutôt que d'histoire sociale, c'est d'histoire économico-sociale qu'il faudrait presque toujours parler". 3 Michelle Perrot parle ailleurs " "d'une certaine confusion dans les termes et d'un manque de définition et de but", 5 de "quelquechose qui cherche son identité dans une forêt de termes: cultures, mentalités, psychologies collectives, anthropologie, représentation, idéologies, etcétera". ${ }^{6}$

Une autre tendance définit l'histoire sociaie assez précisément. Jean Bouvier: "L'histoire sociâle, nous l'avons déjà dit et répété, et je dois le

2 Paris, P., U. F, 1967.

3 Ibid, p. 4J.

4 "The Strengths and Weaknesses of French Social History", dans Journal of Social History, hiver 1976, vol. 10, num. 2, p. 166.177

5 Ibid, p. 166.

albid, p. 171. 
répéter, étudie de grands ensembles: les classes, les groupes sociaux, les catégories socio-professionnelles". ${ }^{\top} \mathrm{Ou}$, encore plus clairement, Ernest Labrousse: Marc Bloch, Lucien Febvie, Georges Lefebvre, Henri Sée, Henri Hauser... "considéraient l'histoire sociale, c'est-à-dire l'histoine des groupes sociaux et de leurs rapports, comme un secteur central de l'his. toire". $\mathrm{s}$

Enfin, une troisième tendance, loin d'y voir "un secteur" de l'histoire comme Labrousse, élargit la notion d'"histoire sociale" à un tel point qu'elle devient identique avec la notion même d'histoire. Ainsi Pierre Goubert: "Pour moi, histoire voulait toujours dire histoire sociale", 9 ou Pierre Vilar: "Je regrette un peu, pour parler franchement, le titre donné à notre rencontre, en ce sens que je n'imagine pas une histoixe qui ne serait pas sociale, ni un domaine social qui ne serait pas histo. rique". ${ }^{10}$

Si l'on voulait bien admettre que pour des raisons différentes, ceux qui refusent à l'histoire sociale un domaine propre rejoignent ceux qui étendent son domaine au point de lui accorder toute la terre, alors nous trouvons en réalité en présence de deux tendances: une, majoritaire, qui dispute à l'histoire sociale ses titres épistémologiques, et celle qui lui accorde comme objet ou domaine "l'histoire des groupes sociaux et de leurs rapports".

Qu'il me soit permis, du moins provisoirement, de ne pas donnex une opinion sur ce différend et d'essayer par contre d'utiliser l'observation précédente pour le contexte qui nous préoccupe ici.

Je dirai : si l'"histoire sociale" c'est "l'histoire des groupes sociaux et de leurs rapports", qu'est-ce que "l'histoire sociale de l'art"? Je pose la question avec d'autant plus d'inquiétude que les numéros $4 / 5$ et 6 de la revue de notre petit groupe, Histoire et Critique des Aris, ont été inclus par quelqu'un qui préparait le CAPES dans une liste envoyée de Toulouse à la Librairie la Hune avec la précision suivante: "Histoire et Critique des Arts (revue de l'histoire sociale de l'art)". Personnellement je ne savais pas que nous faisions de l'histoire sociale de l'art, je pensais même qu'un petit texte qu'on imprimait toujours sur la couver. ture évitait une telle interprétation. Raison de plus donc de me deman der: L'histoire sociale de l'art qu'est-ce que c'est?

7 L'Histoire Sociale, op cit, p. 239.

8 lbid., p. 2 .

${ }_{9}$ Ibid, , p. 97.

10 Ibid, p. $44-45$ 
Je constate d'abord que "l'histoire sociale de l'art" n'est pas la fille de "l'histoire sociale". Elle n'est pas le fruit ou le résultat de l'influence exercée par la reflexion des historiens sur des historiens d'art. Là effectivement les ponts sont coupés depuis la fin du 19e siècle. Chez Hauser par exemple ce sont les philosophes (Dilthey) ou les historiens d'art (Devořak) qui l'ont marqué mais pas les historiens, malgré ses études auprès de Troeltsch. A vrai dire, je ne connais pas un seul ouvrage d'un historien d'art prétendant faire de l'histoire sociale de l'art chez lequel on pourrait discerner l'influence directe de la reflexion d'une école historique. Par contre les exemples d'histoxiens, proches ou descendants directs de l'école des Annales et du courant de l'histoire sociale, qui traitent des sujets pris du domaine traditionellement réservé à l'histoire de l'art, sont plutôt nombreux (M. Agulhon, M. Vovelle, G. Duby, C. Ginzburg etc.). Je crois donc que nous devons abandonner cette piste et nous adresser directement aux auteurs d'ouvrages d'histoire sociale de l'art pour trouver une réponse à notre question : qu'est-ce que l'histoire sociale de l'art, ou, plus précisément, l'histoire sociale de l'art a-t-elle un objet propre, distinct de celui de l'histoire de l'art?

J'oserais dire qu'aucun des auteurs qui ont écrit sur l'histoire sociale de l'art ne repond à une telle question. Enrico Castelnuovo, qui affirme dans son plaidoyer en faveur de l'histoire sociale de l'art ("Per una storia sociale dell'arte") qu" il faudrait avant tout distinguer 'l'histoire sociale de l'art' de la 'sociologie de l'art' ", 11 non seulement ne se donne pas la peine de justifier une telle distinction, mais, en plus, reprend entièrement me semble-t-il, les définitions de Francastel quant à l'objet de la sociologie de l'art. Ainsi il consacre la moitié de son article ${ }^{12}$ à un "Bilan des recherches récentes" en suivant une division "typologique" des objets d'étude de l'histoire sociale de l'art : "Commanditaires, public, institutions, artistes, oeuvres". N'est-ce pas, en plus resireint, le principe qui préside à l'énumération des objets d'étude de la sociologie de l'art selon Pierre Francastel? Dans son article "Art et Sociologie", publié dans L'Année Sociologique en 1949,13 ce dernier énumére "les voies qui ouvrent un large champ à la sociologie de l'art" et il cite: "les artistes dans la société"; "Art et public"; "Mécènes et artisans";

11 P. 65 de la versión française "L'histoire sociale de l'art - un bilan provisoixe", dans Actes de la Recherche en Sciences Sociales, num. 6, décembre 1976.

12 Ibid., p. 69.75

13 L'Année Sociologique, Troisième Série: 1940-1948, tome II, Paris, P, U, F, 1949, p. 491-527. 
"Art et techniques"; "Art et langage"; "Groupes, art et classes"; "Thèmes et symboles sociaux"; Formes et société".

Par ailleurs on peut se demander en quoi l'étude des commanditaires, du public, des institutions, des artistes ou des oeuvres ne ferait pas partie du domaine de l'histoire de l'art? Comment François Benoît ou Léon Ronsenthal, Willem Martin ou Hans Floerke qui ont toujours étudié ces facturs avec attention, cesseraient-ils d'être des historiens d'art toutà-fait "classiques" et par quel coup de baguette magique ils se transformeraient en historiens sociaux de l'art?

Prenons le livre de $\mathrm{T} . \mathrm{J}$. Clark, Image of the People et lisons son chapitre programmatique intitule "On the Social History of Art". 14 Pouvons-nous dire après sa lecture que nous possedons une délimitation, même vague, de l'histoire sociale de l'art et de son objet? Je dirais que nous avons un excellent plaidoyer en faveur d'une autre histoire de l'art, mais l'histoire sociale de l'art comme quelque chose de distinct de l'histoire de l'art, où voyez-vous cela? Reprenons rapidement, poux ne pas être injustes, les principales thèses de Clark:

- "This book sets out.. to discover the actual, complex links which bind together art and politics in this period"; ${ }^{15}$

- "It tries to reconstruct the conditions in which art was, for a time, a disputed, even an effective part of the historical process"; ${ }^{16}$

- If the social history of art has a specific field of study, it is exactly this - the processes of conversion and relation, which so much art history takes for granted". ${ }^{17}$

- "The social history of art sets out to discover the general nature of the structures that he [the artist] encounters willy - nilly; but it also wants to locate the specific conditions of one such meeting"; 19

- "What use did the artist make of pictorial tradition? (...) when one writes the social history of art one is bound to see it in a different light; one is concerned with what prevents representation as much as what allows it; one studies blindness as much as vision", 19

14 Thames and Fudson, London, 1979, p 9-20.

15 Ibid., p. 10 .

16 rbid.

17 Ibid., $\mathrm{p}$. 12.

18 Ibid, p. 13.

19 Ibid, p. 15 
Et finalement:

- "I have been arguing for a history of mediations, for an account of their change and ambiguity. What this means in practice may become clearer if I tie it down to some familiar problems of art history". 20

- "I have been saying that there can be no art hisiory apart from other kinds of history". 21

Ces passages sont suffisamment clairs me semble-t-il pour éviter d'énu. mérer aussi ce qui, selon Clark, serait une histoire sociale de l'art mal comprise (les oeuvres d'art comme refléts d'idéologie, des rapports sociaux, de l'histoire; l'histoire comme "arrière-plan" de l'oeuvre d'art: les analogies intuitives entre forme et contenu).

Restons-en aux développements positifs de Clark: quel historien d'art qui ne soit pas un disciple de l'oeuvte tardive de Wölflin, quel historien d'art qui ne croit pas à l'autogénèse des formes, quel historien d'art qui ne soit pas, lâchons le mot, un formaliste à outrance ne considérerait pas que les objectifs de Tim Clark sont des objectifs tout à fait légitimes de l'histoire de l'art, au moins pour la période envisagée, puisqu'on accorde aux XVIIIe et XIXe siècles, n'est-ce pas, des libertés tout à fait exceptionnelles? 22 Je crois que Tim Clark lui-même, s'il était présent ici aurait repondu à cette question par l'affirmative. D'ailleurs n'oppose-t-il pas ouvertement à un moment sa conception de l'histoire de l'art à ce qu'il appelle "an empirical notion of the social history of art", en entendant par là "the immediate conditions of artistic production and reception: patronage, sales, criticism, public opinion"? ${ }^{23}$

\section{VI}

Je crois que ces exemples, pris parmi les textes majeurs qui font son

20 Ibid, p. 13

21 Ibid., p. 18.

22 "For other socially oriented approaches to the period, which is particularly conducive to Marxist interpretation, see.." (Robert Rosenblum, Transformations in Late Eighteenth Cenutry Art, Princeton University Press, Princeton, 1967, p. 50) .

${ }_{23}$ T. J Clark, Image of the People, op. cit., p. 11. 
apologie, suffisent pour nous convaincre du fait que "l'histoire sociale de l'art" n'est pas une discipline indépendante avec un objet ou domaine propres. Du moins elle ne l'a pas été jusqu'ici. Elle pourra, peut-ètre, le devenix, je n'en sais rien.

Pour le moment, ce qui se cache derrière l'appellation "histoire sociale de l'art" ce n'est pas un domaine mais une orientation méthodologique ou interprétative, différente de celle qui prédomine (ou du moins prédominait-elle) à l'intérieur de l'histoire de l'art; c'est un plaidoyer en faveur d'une "autre histoire de l'art" comme l'écrivait si justement Castelnuovo au début de son article à propos des Remarks on the Method of Art-History, publiés par Antal en 1949, après la débâcle de la réception de Florentine Painting par les critiques. ${ }^{24}$

Une orientation méthodologique différente à l'intérieur de l'histoire de l'art: voilà ce que vois, ce que je sens et ce que je comprends en lisant les ouvrages sur l'histoire sociale de l'art ou d'histoire sociale de l'art.

D'ailleurs, et ce sera ma dernière pièce à l'appui de cette argumentation, n'est-ce pas quand même formidable que Arnold Hauser, critiqué pour les passages excessivement courts sur le manièrisme dans son Histoire sociale de l'art et de la littérature, voulant de surcroît mettre sa méthode à l'épreuve de l'analyse détaillée d'un phénomène artistique, ait écrit un fort volume sur le Maniérisme dans l'introduction duquel il déclare sans ambages que la matière d'une histoire de l'art "pure" ne suffisait pas pour la compréhension du phénomène dans la mesure où l'on conçoit l'histoire de l'art comme partie de l'histoire de la culture? Que les vrais problèmes ne pouvaient être résolus "que dans le contexte d'une problématique de l'histoire de l'esprit"? ${ }^{25}$

24 "Il y a environ vingt-cinq ans, en 1949, le Burlington Magazine ouvrait ses colonnes à un long plaidoyer de Frederick Antal en faveur d'une 'autre' histoire de l'art" ("L'Histoire sociale de l'art", op. cit, p. 63).

25 Annold Hauser, Der Manierismus, Verlag C. H. Beck, Munich, 1964, p. v-vi: "Linen besonderen Hinweis dürfte jedoch die Eigenart der befolgten Methode erfordexn. Sie ist eine durchaus historische, doch keine schlechthin kunsthistorische; mit einer solchen wäre die zu lösende Aufgabe überhaupt nicht in Angriff zu nehmen gewesen. Eigentlich kann keine komplexere und umfassendere, die Geschichte der Kunst als Teil des Kulturprozesses betreffende Frage lediglich auf Grund des jeweiligen kunsthistorischen Materials und mittels einer auf dieses Material zugeschnittenen Methode geklärt werden; solche Fragen lassen sich nur im Rahmen eines geistesgeschichtilichen Problemzusammenhangs und mit Hilfe einer weit vielseitigeren methodologischen Einstellung, als die der reinen Kunstgeschichte ist, sinnvoll stellen und befriedigend beantworten." 
L' histoire sociale de l'art est-ce alors un habit neuf à une vieille idée, à un vieux problème qui hante les historiens d'art depuis longtemps (et il est effectivement très difficile d'échapper à cette hantise), à savoir le problème de l'interprétation autant que l'insistance sur l'importance de quelques thèmes négligés par l'histoir e de l'art traditionnelle et conservatrice (les commanditaires, le public, les institutions)? Je dirais "oui" sans hésitation.

Un vieux problème. Quand même pas très, très vieux. Puisqu'il est inséparable de la spécialisation, de la constitution de l'histoire de l'art comme discipline indépendante. Le problème ne se pose pas pour Winqckelmann, qui, tout en faisant l'analyse des formes la plus remarquable, nous parle autant du climat, des moeurs et de la politique dans Ia cité.

Le problème se pose à partir de la deuxième moité du $19 \mathrm{e}$ siècle. On peut à posteriori penser trouver des antécédents et des ancètres avant l'époque de l'ascension triomphante de la bourgeoisie, mais cela est-ce vraiment convaincarit?

Je prendrai un seul exemple, tiré du Grand lizre des peinires de Gérard de Lairesse, traduction française de 1787:

On ne peut douter que chaque artiste ne s'applaudisse de son choix, qu'il regarde comme le meilleur et qu'il ne cherche véritablement à s'y distinguer: et en effet, les bambochades et les sujets de la nature commune ne demandent pas moins de talent dans l'éxécution pratique, que les grands sujets tirés d'Homère, de Virgile ou de l'histoire; quoiqu'il soit plus facile, à la vérité, d'exécuter des choses qu'on a tous les jours sous la vue, que celles qu'on ne voit, pour ainsi dire, que par les yeux de l'esprit, qui doit se représentex, par comparaison, les événements dont on a entendu faire le récit, ou qu'on a lus dans l'histoire. Voilà aussi la raison pourquoi Rubens et Van Dyck, qui vivoient dans le grand monde, ont arrêté leurs regards sur la partie la plus sublime de l'art; tandis que Jordans et Rembrandt ont adopté ce qu'on appelle proprement Peinture de genre, et que le Bamboche et Brouwer ont montré un goût bas et commun, chacun suivant les idées qu'ii avoit puisées dans la classe d'hommes qu'il fréquentoit. ${ }^{20}$

26 Gérard de Lairesse, Le grand liwre des peintres ou l'art de la peinture, Pais, I787, tome I, p. 296-297. Les libertés prises dans la traduction française et sa comparaison avec le texte original hollandais publié du vivant de Géraxd de Lairesse, devraient faire l'objet d'une étude à part. Je me contente ici de signaler que là où Lairesse dit "art bourgeois" le tradue eur met "peinture de genre" et de citer le texte original: "Maax gemakkelyker valt het voor een Burger, een burgerlyke xol dan een andere te 
Qu'avons-nous ici? Une histoire sociale de l'art à l'état pratique? Une théorie du reflèt (une théorie qui malgré l'obstination de certains qui veulent la rattacher au marxisme mécanique est indissociable des débuts et de la montée de la classe bourgeoise) qui exige une histoire sociale de lart comme complément? Un programme (non réalisé) d'une étude comparative entre l'art de trois classes sociales, préfigurant ainsi la définition de Labrousse et l'adaptant à l'histoire de l'art? Peut-être pourrionsnous faire effectivement des généalogies pareilles, mais est-ce qu'on gagne grand'chose en faisant cela? Je crois en effet que c'est à partir de la seconde moitié du 19e siècle que les problèmes que recouvre "l'histoire sociale de l'art" prennent vraiment forme et commencent à jouer un rôle.

C'est-à-partir de ce moment là, une fois que le dogme de l'histoire de l'art comme une histoire interne des formes a été mis en place, que se fait jour une multitude de tentatives de faire autre chose. Surtont: 1) de concevoir l'histoire de l'art comme partie intégrante d'un ensemble plus grand; 2) de faire appel à des éléments "extérieurs" aux oeuvres pour expliquer ces dernières.

Que se soit "l'histoire de la culture" (la Kulturgeschichte) de Jacob Burckhardt ou son mélange avec les théories d'Hippolyte Taine (pensez a Justi, pensez, puisque nous sommes dans l'année commémorative de Raphaël, au Raphaël, sa vie, son oewvre et son temps d'Eugène Müntz de 1881, lisez son chapitre IX "Raphaël à Rome - La ville éternelle au commencement du seizième Siècle - Jules II et la cour pontificale: prélats, humanistes, grands seigneurs et banquiers - Le monde des artistes", quj couvre cinquante pages imprimées in quarto où le nom de Raphaël apparaît de temps en temps, comme s'il fallait rassurer le lecteur qu'il était bien entrain de lire une monographie sur Raphaël et non pas une sur Michelange ou Bramante); que ce soit "la science de la culture" (la Kulturwissenschaft) de Aby Warburg avec ses répercussions jusqu'à notre après guerre grâce à la réception de Studies in Iconology; que ce soit "l'histoire de l'art comme histoire de l'esprit" (la Geistesgeschichte) de

speelen; alzo ook roor een Schilder, te blyven by het verbeelden van het geen hem dagelyks vookkomt: dewyl onze herssenen zyn als een glaze bol, in 't midden van een kamer opgehangen, welke door alle voorwerpen, die zich vertoonen, aangedaan word, ch een indruk daar van behoud. Zo zag men Rubbens en van Dyk, mannen die dagelyks te Hoof en by de Grooten verkeerden, hunne gedachten op het verheevene der Konst vesten; Jordaans en Rembrant weder op het burgerlyke; Ramboots en Bruwer op het allergeringste: en dus ieder na de maat hunner neigingen, voor zo veel dezelve tot den ommegang met menschen van hunne soorten strekten " (Gerard de Lairesse, Het Groot schilderboek, Amsterdam, 1707, tome I, p. 185.) 
Max Dvořak auprès duquel ont étudié à la fois Antal et Hauser); toutes ces écoles, toutes ces tendances, au-delà de leurs différences et de leurs divergences, n'ont-elles pas en commun la certitude a) qu'une histoire de l'art entendue comme une stricte "histoire des formes" aboutirait à une tautologie; $b$ ) que pour apprécier, comprendre, expliquer soit des phases de cette histoire, soit des oeuvres d'art individuelles il fallait faire appel à d'autres objets, à d'autres monuments, à d'autres faits qu'aux seuls objets, monuments ou faits artistiques?

Lequel des objectifs affichés de l'histoire sociale de l'art ne se trouveraił. il pas formulé et d'une certaine manière réalisé par les protagonistes de ces trois courants, constamment dénigrés et critiqués par l'histoire de l'art "proprement dite", à savoir par l'histoire de l'art dominante?

Michael Baxandall, qui travaille à l'Institut Warburg de Londres, me disait il y a un mois qu'il ne se concevait pas comme un historien social de l'art ("social historian of art") mais comme un "cultural historian". A ma question pourquoi alors son livre sur la peinture italienne du $15 \mathrm{e}$ siècle portait le sous-titre "manuel de base pour l'histoire sociale des styles picturaux" ("a primer in the social history of pictorial style") il m'a répondu que ceci était plutôt destiné à rendre les historiens d'art un peu mal à l'aise qu'à être pris au sérieux.

N'est-ce pas un autre indice sur la piste qui nous amenait à dire que l'histoire sociale de l'art est en réalité un prête-nom pour autre chose, à savoir des tendances qui ont existé auparavant sous des noms différents?

Je pense à une phrase de Monsieur Jacques Thuillier, employée lors de sa leçon inaugurale au Collège de France le 13 janvier 1978, et dirigée contre ceux qui nient qu'il y ait une hiérarchie de "valeurs", de "qualités" artistiques ou esthétiques valables hors du temps et de l'espace, hiérarchie de valeurs/critères qui permettrait par exemple de "mesurer" une fois pour touter la qualité de Poussin: "L'histoire de la peinture du XVIIe siècle n'est pas l'histoire de tous les panneaux, toiles et murs couverts de peinture au XVII siècle. Pour l'historien d'art, Poussin et Le Sueur l'emportent dix fois sur Baugin, lequel l'emporte de loin sur Dudot, et Dudot plane au-dessus d'un amas informe, qui ne fait plus partie de l'objet de l'Histoire de l'art, qui concerne seulement la recherche comme les papiers d'archives ou les mémoires de l'époque, à titre de repère et document. Je sais bien", dit Jacques Thuillier, "que d'aucuns voudraient faire croire l'inverse : mais c'est précisément qu'ils ne sont pas 
des historiens d'art, mais simplement des historiens s'occupant de l'art. Ils s'intéressent à une production et non à une création". 27

Cette dernière phrase si caractéristique pour l'histoire de l'art traditionnelle et conservatrice, la voilà répétée mille fois, au nom de l'histoire de l'art "proprement dite". Combien de fois ne l'a-t-on pas utilisée contre les "généralisations" de Burckhardt et de Dvořak ou contre les travaux "littéraires" de Warburg et de son école?

Mais on peut aller plus loin et dire que ces reproches se sont échangés aussi entre les dissidents de l'histoire de l'art orthodoxe. Je cite par exemple Warburg critiquant Burckhardt, pour lequel il avait néanmoins une admiration sans bornes : bien que "pionnier exemplaire" de la science, Burckhardt "au lieu d'affronter le problème de l'histoire de la culture [de la Renaissance] dans la totalité de son attraction artistique, il l'a découpé en parties sans liens entre elles [le Cicerone et la Kultur der Renaissance], et les a analysées et décrites l'une après l'autre d'une manière souveraine". 28

Je cite par exemple Dvorak attaquant en 1914 les responsables d'une "grave confusion qui commence à menacer de nouveau l'histoire de I'art", ${ }^{29}$ et il suffit de lire son texte pour comprendre qu'un des principaux coupables de cette confusion c'est Aby Warburg: "Si alors l'histoire de l'art est, conformement à son essence et à ses tâches, une science historique, elle prend néanmoins une place indépendante dans le cadre des disciplines historiques. Sa tâche est "la recherche et la description [Darstellung] de tous les faits qui permettent de discerner le développement du vouloir artistique humain [menschliches Kunstwollen], dans leurs rapports de causalité" (Tietze). Et ce qui n'est pas rattachè directe. ment à cette tâche, se trouve à l'extérieur de son domaine. Ceci vaut surtoit pour l'histoire de la culture, avec laquelle l'histoire l'art a été souvent confondue. (...) Le plus grand progrès de l'histoire de l'art durant les dernières décennies résidait précisément dans le fait ... qu'elle

27 Jacques Thuillier, leçon inaugurale faite le vendredi 13 janvier 1978, Collège de France, chaire d'histoire de la création artistique en France, Collège de France, 1978, p. 18

28 A. Warburg, "Bildniskunst und florentinisches Bürgertum", dans Gesammelte Schriften, Teubner, Leipzig/Berlin, 1932, tome I, p. 93"

29 Max Dvorak, "Über die dringendsten methodischen Erfordernisse der Erziehung zur kunstgeschichlichen Forschung", dans Die Geistesvissenschaften, cahier 34, 21 mai 1914, p. 932. 
a elevée l'observation stilistique au point d'être la source la plus impor. tante de la critique et de l'explication artistique". ${ }^{30}$

Je cite Francastel qui, dans "Problèmes de la sociologie de l'art", écrivait à propos de l'Institut et de l'école de Warburg: "Malheureusement, il s'est progresivement laissé dominer par une conception de plus en plus philosophante de l'esthétique. Si l'étude des oeuvres d'art ne peut être conçue comme une pure histoire des formes, elle ne peut non plus être envisagée comme une province de l'histoire des idées". ${ }^{31}$

Et ainsi de suite.

\section{VIII}

Je viens de parler de l'histoire sociale de l'art comme prête-nom d'une orientation méthodologique minoritaire à l'intérieur de l'histoire de l'art, poursuivant tantôt les objectifs de l'histoire de l'art entendue comme partie de l'histoire de la culture, tantôt ceux de l'histoire de l'axt comme élément de la science de la culture, un xôle joué jusqu'ici d'ailleurs et avec le même succès ambigu, par la "sociologie de l'art.

Mais j'ai laissé consciemment de côté un autre phénomène qui me parait aussi très important: l'histoire sociale de l'art est souvent entendue comme synonyme de l'histoire de l'art marxiste.

Cela peut étonner, après tout ce qui vient d'être dit. Pourtant il ne fait pas de doute à mes yeux que tant sur le plan des personnes que sur celui des ensembles plus grands, l'identification est courante.

Ainsi, daris un colloque sur "Burckhardt et la Renaissance cent ans après", organisé par l'Université de Kansas à 1960, Klaus Berger défendait Jacob Burckhardt devant les critiques de... Arnold Hauser qu'il qualifiait de critique Marxiste. ${ }^{32}$ Ce quiproquo, alimenté par des développements récents à l'intérieur de la tradition marxiste et communiste, autant qu'à l'intérieur de la discipline de l'histoire de l'art, a pour ainsi dire des bases trop solìdes pour disparaitre de si tôt. Je veux comme preuves 1) le fait que les écrits de Hauser lui-même, qui infirment une telle identification, ne parviennent pas à stopper la diffusión de ce

$30 \mathrm{Ibid}, \mathrm{p}, 935$ et caheir 35,28 mai 1914, p. 960

81 "Problèmes de la sociologie de l'aut" dans Traité de Sociologie, publié sous la direction de Georges Gurvitch, Paris, P. U. F., 1960, troisième édition 1968, p. 280

32 Klaus Berger, "Jacob Burckhardt as an art historian", dans Jacob Burckhardt and the Renaissance a hundred years after, The University of Kansas, Lawrence, 1960, p. 41 . 
mythe; 2) le fait que la problématique de Hauser a été critiquée de ce point de vue d'une manière assez perspicace par des auteurs se réclamant de la tradition marxiste, comme par exemple Peter Klein en Allemagne de l'Ouest ${ }^{33}$ ou Laszlo Barlay en Allemagne de l'Est, ${ }^{34}$ sans que cela serve à quelque chose non plus.

Il faut le dire: cette identification de l'histoire sociale de l'art avec l'histoire de l'art marxiste est l'oeuvre aussi bien d'adversaires que de défenseurs de l'histoire sociale de l'art "Plus: de défenseurs d'une cer. taine compréhension de marxisme lui-même.

Une telle identification entre "Histoire sociale de l'art" et "histoire de l'art marxiste" était, par exemple, à la base de la décision du groupe d'historiens d'art des USA "Caucus for Marxism and Art" (qui organisait régulièrement ces dernières années une section dans le cadre du Congrè Annuel de la College Art Asociation) de renoncer à une telle section en 1981 (au Congrès de San Francisco) et de participer à la place dans la section The Social History of Art organisée par Robert Herbert de Yale University. En effet deux parmi les quatre communications qui ont été lues, étaient l'oeuvre de membres du Caucus for Marxism and Art, sans que cela suscite une discussion de principe quelconque.

Ceci est un exemple encore innofensif, bien que significatif d'un état d'esprit. Mais il y a pire. On peut, c'est bien connu, concervoir le marxisme comme un supplément d'âme au système capitaliste existant. Dans le domaine des sciences sociales le marxisme n'est-ce pas un volet additionnel à l'idéologie libérale de l'histoire?

Ainsi nous pouvons lire dans la revue Kritische Berichte, publiée par nos amis de l'Ulmer Verein, assaciation d'historiens d'art en Allemagne Fédérale, sous la plume de Martin Warnke, vétéran fatigué des "révol. tes" de 68, qui représente le progressisme intitutionnalisé dans ce pays, que les historiens d'art conservateurs "qui s'élèvent contre un élargissement du domaine de l'histoire de l'art en direction de l'histoire sociale ("eine sozialgeschichtliche Erweiterung des kunstgeschichtlichen Gegens. tandfeldes") n'ont pas su profiter des possibilités qui leur ont été offer tes"., 35

33 Peter Klein, "Arnold Hausers Theorie der Kunst", dans Kritische Berichte, num.3, 1978, p. 18-27.

34 Laszlo Barlay, "Wissenssoziologie als Kulturgeschichte? Zur Arnold Hausers 'Sozial" geschichte der Kunst und Literatur", dans Sinn und Form, vol. 24, 1972, num. 3, p. $632-644$.

35 Martin Warnke, "Rückruf", dans Kritische Berichte, num. 4, 1976, p. 55. 
Cette réduction du marxisme à une pierre qui pourrait, sans susciter que des xéajustements mineurs, parfaire la mosaîque panoramique de l'histoire de l'art traditionnelle est de plus en plus acceptée, malgré des résistances indiscutables, puisque elle fait de lui une théorie qui contribue effectivement à l'intégration et à la cohésion de l'orđre établi.

\section{IX}

Ce manque de présion concernant la spécificité de l'histoire sociale de l'art, cette série de "malentendus" qui la traversent, ce flou qui l'entoure, permettent en effet un grand nombre d'accomodements en provenance de plusieurs horizons. Ce flou sert effectivement d'alibi à ceux et à celles qui refusent de prendre une position aussi nette que possible devant les problèmes qui divisent à la fois notre discipline et le monde. Michèlle Perrot, parlant de l'histoire sociale, il est vrai, mais je crois que cela est aussi valable pour l'histoire sociale de l'art, évoquait "sa force de séduction à cause de son apparente facilité" et le fait que "tout le monde peut s'en réclamer". ${ }^{36}$ L'histoire sociale de l'art c'est vraiment de la facilité: elle n'a pas d'objet propre, elle n'engage à xien, et l'on peut la pratiquer d'une manière très avantageuse. Les conservateurs, grâce à l'emploi du mot magique "social" sont "couverts" devant toute accusation de conservatisme (bien sûr il y a aussi des conservateurs du type Ulrich Middeldoxf, si à propos cité par Castelnuovo, ${ }^{37}$ qui ne voient toujours pas les avantages ou même la légitimité d'une histoire sociale de l'art: mais je me demande si ce n'est pas la fraction conservatrice qui est entrain de perdre la course). Les historiens d'art libéraux font une oeuvre "progressiste", une oeuvxe "engagée" tout en évitant à la fois les étiquettes incommodes et les conséquences théoriques et pratiques qu'entraînerait une adhesion de principe au marxisme. Les historiens d'art de gauche peuvent à leur tour éviter l'étiquette du marxisme, certainement peu favorable à une carrière, universitaire ou autre, sans renier leurs idées. Il suffirait qu'ils abandonnent le "fétichisme" de quelques mots.

30 Michelle Perrot, "The Strengths and Weaknesses of French Social History" op. cit, p. 166 .

37 Il s'agit du compte-rendu du livre de Michael Baxandhall, Painting and Experience in Fifteenth Century Italy par Ulrich Middeldorf dans le Art Bulletin de juin 1975, cité par Castelnuovo "L'histoire sociale de l'art", op. cit., p. 64. 
En terminant, et après avoir tenté d'arrêter le coupable, permettez-moi de faire son éloge.

Les effets positifs de cette discipline inéxistante qui s'intitule "Histoire sociale de l'art" sont aussi indéniables que ses méfaits. Là où l'histoire de l'art conservatrice règne sans partage l'histoire sociale de l'art ouvre une brêche utile. Elle peut servir de catalyseur, acculer les conservateurs orthodoxes au pied du mur.

Dans une période où l'intolérance monte et la chasse aux sorcières recommence, la position libérale que représente en effet l'histoire sociale de l'art, peut être l'équivalent d'un petit oasis sous les arbres duquel on peut parler calmement de quelques questions brûlantes sans risquer immédiatement sa peau.

Tout cela est vrai, et tout cela n'est pas negligeable. Et pourtant je crois que nous gagnons tous quelque chose en appellant un chat un chat et en affrontant les problèmes de front.

De toute manière "l'histoire sociale de l'art" ne risque pas de disparaître, puisqu'elle correspond à des besoins qui permettent sa repro. duction. La critique de l'histoire sociale de l'art, de ses prétentions et des promesses qu'elle tient sans pouvoir les satisfaire, me paraît une nécessité. Nous n'avons pas besoin de cette confusion entre l'"objet d'une discipline" d'une part et les "méthodes d'analyse de son objet" d'autre part. Les prétendus objets de l'histoire sociale de l'art font partie de droit et de fait des thèmes de 1" "histoire de l'art".

Pax contre le vrai débat autour de la théorie et des méthodes appliquées en histoire de l'art reste à faire; ne serait-ce que pour reprendre les débats antérieures qui ont eu liẹ autour de ces questions capitales.

10 décembre 1983 